\title{
A compact active sound absorption system compensating near-field effect of the secondary source
}

\author{
Jun Wang ${ }^{\text {a) }}$, Jing $\mathrm{Lu}^{\mathrm{b})}$ and Xiaojun Qiu ${ }^{\mathrm{c})}$ \\ (Received: 7 June 2017; Revised: 24 September 2017; Accepted: 5 October 2017)
}

\begin{abstract}
In a compact active sound absorption system where the error sensor is close to the secondary source, the near-field effect of the secondary source deteriorates the system performance severely. This study proposes an improved method by employing an auxiliary calibration process, in which an extra sensor is utilized to obtain relevant impulse responses, and then these impulse responses are used in the control process to eliminate the influence of the near-field effect. Experiments are carried out in a compact active sound absorption system in a duct to demonstrate the feasibility of the proposed method. (C) 2017 Institute of Noise Control Engineering.
\end{abstract}

Primary subject classification: 38.2 ; Secondary subject classification: 35

\section{INTRODUCTION}

Using active noise control to attenuate acoustic reflection is appealing because it can remove bulky passive absorption material needed at low frequencies ${ }^{1-4}$. Apart from the applications in room and duct acoustics, active sound absorption also has potential use in making objects invisible to incident detecting waves ${ }^{5}$.

The pressure release and impedance matching methods have been proposed for absorption control by minimizing the pressure behind the material or making the equivalent impedance in front of the material equal to that of the medium to eliminate the cause of the wave reflection $^{6-9}$. The performance of the pressure release method depends on the properties of the passive material, and two sensors are usually needed in the impedance matching method to predict the reflected wave ${ }^{10}$. In order to eliminate the frequency limitation of the distance between two sensors, Han et al. proposed a method to predict the reflected wave with only one error sensor placed on the reflection surface ${ }^{11}$, which was further extended to a strategy that controls the scattering from a rigid sphere ${ }^{12}$. Unfortunately, an ideal reference signal was used in their system, which is not practical for many applications.

The compact active sound absorption system is preferred in many circumstances due to the space limitation.

a) Key Laboratory of Modern Acoustics and Institute of Acoustics, Nanjing University, Nanjing 210093, CHINA; email: 545919359@qq.com.

b) Key Laboratory of Modern Acoustics and Institute of Acoustics, Nanjing University, Nanjing 210093, CHINA; email: lujing@nju.edu.cn (Corresponding author).

c) Faculty of Engineering and Information Technology, University of Technology Sydney, NSW 2007, AUSTRALIA.
Zhu et al. proposed a wave separation method to obtain both the reference signal and the error signal simultaneously and proved the feasibility of the method by several experiments in a duct ${ }^{13}$. However, the near-field effect of the secondary source affects the wave separation results in a compact control system because the sensors are close to the secondary source. This deteriorates severely the absorption performance of the whole system.

In this study, an improved method with an auxiliary calibration process is proposed. An extra sensor is introduced in the calibration process to obtain the relevant impulse responses, which are used to eliminate the near-field effect in the control. An experimental system is established in a duct to show the near-field effect on the wave separation results and the feasibility of the proposed method.

\section{THEORY}

A compact active sound absorption system in a duct is depicted in Fig. 1, where the waves travel along the $z$-axis and the secondary source is placed at the end of the duct with $z=0$. Two sensors, A and B, are placed close to the secondary source acting as the sensors for the compact active absorber. They are pre-calibrated for amplitude and phase matching. In order to evaluate the acoustic absorption performance, two additional sensors, $\mathrm{C}$ and $\mathrm{D}$, are placed farther away from the end of the duct to measure the sound absorption coefficients using the transfer function method ${ }^{14}$. Position $\mathrm{E}$ is the midpoint location for sensors $\mathrm{A}$ and $\mathrm{B}$.

Assuming that the primary source is located in the farfield, the incident and reflected waves by the termination end with the compact active absorber are separated by using the integration method as ${ }^{15}$ : 
The compact active sound absorption system

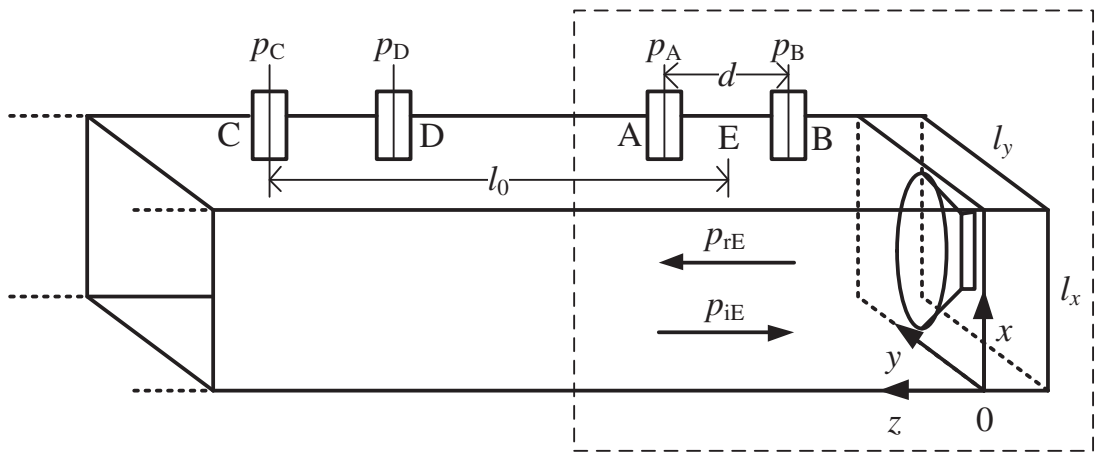

Fig. 1-The compact active sound absorption system.

$$
\begin{aligned}
p_{\mathrm{iE}}(t)= & \frac{1}{4}\left[p_{\mathrm{A}}(t)+p_{\mathrm{B}}(t)\right] \\
& +\frac{c}{2 d} \int_{0}^{t}\left[p_{\mathrm{A}}(\tau)-p_{\mathrm{B}}(\tau)\right] d \tau, \\
p_{\mathrm{rE}}(t)= & \frac{1}{4}\left[p_{\mathrm{A}}(t)+p_{\mathrm{B}}(t)\right] \\
& -\frac{c}{2 d} \int_{0}^{t}\left[p_{\mathrm{A}}(\tau)-p_{\mathrm{B}}(\tau)\right] d \tau,
\end{aligned}
$$

where $p_{\mathrm{iE}}(t)$ and $p_{\mathrm{rE}}(t)$ are the incident and reflected waves at position $\mathrm{E}$, respectively; $p_{\mathrm{A}}(t)$ and $p_{\mathrm{B}}(t)$ are the signals captured by sensors A and B, respectively; $c$ is the speed of the sound in the air; and $d$ is the distance between sensors A and B. For a digital system with a sampling frequency of $f_{s}$, the above integrations can be calculated approximately with summation and many numerical methods such as the trapezoid method can be used for the calculation $^{15}$. For example:

$$
\begin{aligned}
p_{\mathrm{iE}}(n)= & \frac{1}{4}\left[p_{\mathrm{A}}(n)+p_{\mathrm{B}}(n)\right] \\
& +\frac{c}{2 d f_{s}} \sum_{i=0}^{n}\left[\frac{p_{\mathrm{A}}(i-1)-p_{\mathrm{B}}(i-1)}{2}\right. \\
& \left.+\frac{p_{\mathrm{A}}(i)-p_{\mathrm{B}}(i)}{2}\right], \\
p_{\mathrm{rE}}(n)= & \frac{1}{4}\left[p_{\mathrm{A}}(n)+p_{\mathrm{B}}(n)\right] \\
& -\frac{c}{2 d f_{s}} \sum_{i=0}^{n}\left[\frac{p_{\mathrm{A}}(i-1)-p_{\mathrm{B}}(i-1)}{2}\right. \\
& \left.+\frac{p_{\mathrm{A}}(i)-p_{\mathrm{B}}(i)}{2}\right] .
\end{aligned}
$$

For an active sound absorption system, $p_{\mathrm{iE}}(n)$ is regarded as the reference signal and $p_{\mathrm{rE}}(n)$ is the sound to be attenuated. The FxLMS algorithm is implemented to attenuate the separated reflected wave with the separated incident wave acting as the reference signal ${ }^{16}$.

The weakness of the wave separation method using Eqns. (3) and (4) is that plane wave propagating along $z$-axis is assumed in the duct. Unfortunately, the assumption does not hold for compact systems, where high-order modal evanescent waves exist at positions $\mathrm{A}$ and $\mathrm{B}$ because they are in the near-field of the secondary source. The sound pressure in the duct with high-order modes can be described as ${ }^{17}$ :

$p(x, y, z, t)=\sum_{n_{x}=0}^{\infty} \sum_{n_{y}=0}^{\infty} A_{n_{x} n_{y}} e^{-\alpha_{n x n} z} \cos \frac{n_{x} \pi}{l_{x}} x \cos \frac{n_{y} \pi}{l_{y}} y e^{j \omega t}$,

where $\alpha_{n_{x} n_{y}}=\sqrt{\left[\left(n_{x} / l_{x}\right)^{2}+\left(n_{y} / l_{y}\right)^{2}\right] \pi^{2}-(\omega / c)^{2}} ; l_{x}$ and $l_{y}$ are the length and width of the cross section of the duct, respectively; $x$ and $y$ are the axes parallel to the side of $l_{x}$ and $l_{y}$, which are perpendicular to the $z$-axis; $\left(n_{x}, n_{y}\right)$ is the mode of the wave with the initial amplitude $A_{n_{x} n_{y}}$; and $\omega$ is the angular frequency. For example, in a $0.17 \mathrm{~m} \times 0.17 \mathrm{~m}$ duct, if the amplitude of $(1,0)$ or $(0,1)$ mode from the secondary source at $z=0$ is assumed to be 1.00 at $800 \mathrm{~Hz}$, it attenuates to 0.57 at $0.05 \mathrm{~m}$ and to $1.52 \times 10^{-6}$ at $1.15 \mathrm{~m}$ away from the secondary source. These high-order modes affect the signals picked up by the sensors significantly in the compact system.

\section{METHOD}

To eliminate the influence of the near-field effect, an additional calibration process is proposed prior to the control process. Exciting the secondary source with a narrow-band noise whose frequencies are below the cut-off frequency of the duct, the impulse response $\left\{w_{\mathrm{SA}}(n), n=1,2, \cdots\right.$, $\left.L_{\mathrm{SA}}-1\right\}$ between the secondary source and $p_{\mathrm{A}}(n)$, and the impulse response $\left\{w_{\mathrm{SB}}(n), n=1,2, \cdots, L_{\mathrm{SB}}-1\right\}$ between the secondary source and $p_{\mathrm{B}}(n)$ can be estimated 
by commonly used adaptive algorithms such as the LMS algorithm $^{16}$.

Assuming that the amplitudes of the evanescent waves are sufficiently small at position $\mathrm{C}$ and the primary source is located in the far-field, the wave due to the $(0,0)$ mode at position $\mathrm{C}, p_{\mathrm{C}(0,0)}(n)$, is approximately equal to $p_{\mathrm{C}}(n)$. With the distance between positions $\mathrm{C}$ and $\mathrm{E}$ being $l_{0}$, the time delay between $p_{\mathrm{C}(0,0)}(n)$ and the wave due to the $(0,0)$ mode at position $\mathrm{E}, p_{\mathrm{E}(0,0)}(n)$, is $\tau=l_{0} / c$. The $p_{\mathrm{E}(0,0)}(n)$ can be calculated as:

$$
p_{E(0,0)}(n)=p_{C(0,0)}\left(n+\tau f_{s}\right)=p_{C}\left(n+\tau f_{s}\right) .
$$

Set an integer number $M$ larger than $\tau f_{s}$, and $\left\{w_{M-\tau f s}(n)\right.$, $\left.n=0,1, \cdots, L_{\tau}-1\right\}$ as an impulse response with a delay of $M-\tau f_{s}$. Note that if $M-\tau f_{s}$ is fractional, $\left\{w_{M-\tau f s}(n)\right\}$ can be calculated with the Lagrange interpolation method ${ }^{18}$. Then $p_{\mathrm{C}}\left(n+\tau f_{s}\right)$ can be calculated by passing $p_{\mathrm{C}}(n+M)$ through $\left\{w_{M-\tau f s}(n)\right\}$ as:

$$
\begin{aligned}
p_{\mathrm{C}}\left(n+\tau f_{s}\right) & =p_{\mathrm{C}}\left[n+M-\left(M-\tau f_{s}\right)\right] \\
& =\sum_{l=0}^{L_{\tau}-1} w_{M-\tau f_{s}}(l) p_{\mathrm{C}}(n+M-l) .
\end{aligned}
$$

After calculating $p_{\mathrm{E}(0,0)}(n)$, the equivalent impulse response $\left\{w_{\mathrm{SE}(0,0)}(n), n=0,1, \cdots, L_{\mathrm{SE}(0,0)}-1\right\}$ between the secondary source and $p_{\mathrm{E}(0,0)}(n)$ can then be estimated by the LMS algorithm ${ }^{16}$.

In the controlling process, both the primary source and the secondary source $s(n)$ are excited simultaneously.
The sound pressure related only to the primary source at positions $\mathrm{A}$ and $\mathrm{B}$ can be expressed as:

$$
\begin{aligned}
& p_{\mathrm{A}}^{\mathrm{p}}(n)=p_{\mathrm{A}}(n)-\sum_{l=0}^{L_{\mathrm{SA}}-1} w_{\mathrm{SA}}(l) s(n-l), \\
& p_{\mathrm{B}}^{\mathrm{p}}(n)=p_{\mathrm{B}}(n)-\sum_{l=0}^{L_{\mathrm{SB}}-1} w_{\mathrm{SB}}(l) s(n-l) .
\end{aligned}
$$

The incident and reflected waves related only to the primary source at position $\mathrm{E}$ can be calculated similarly as that in Eqns. (3) and (4) by:

$$
\begin{aligned}
p_{\mathrm{iE}}^{\mathrm{p}}(n)= & \frac{1}{4}\left[p_{\mathrm{A}}^{\mathrm{p}}(n)+p_{\mathrm{B}}^{\mathrm{p}}(n)\right] \\
& +\frac{c}{2 d f_{s}} \sum_{i=0}^{n}\left[\frac{p_{\mathrm{A}}^{\mathrm{p}}(i-1)-p_{\mathrm{B}}^{\mathrm{p}}(i-1)}{2}\right. \\
& \left.+\frac{p_{\mathrm{A}}^{\mathrm{p}}(i)-p_{\mathrm{B}}^{\mathrm{p}}(i)}{2}\right] \\
p_{r \mathrm{E}}^{\mathrm{p}}(n)= & \frac{1}{4}\left[p_{\mathrm{A}}^{\mathrm{p}}(n)+p_{\mathrm{B}}^{\mathrm{p}}(n)\right] \\
& -\frac{c}{2 d f_{s}} \sum_{i=0}^{n}\left[\frac{p_{\mathrm{A}}^{\mathrm{p}}(i-1)-p_{\mathrm{B}}^{\mathrm{p}}(i-1)}{2}\right. \\
& \left.+\frac{p_{\mathrm{A}}^{\mathrm{p}}(i)-p_{\mathrm{B}}^{\mathrm{p}}(i)}{2}\right] .
\end{aligned}
$$

The sound pressure due to the $(0,0)$ mode contributed only by the secondary source at position $\mathrm{E}$ can be expressed as:

\section{Table 1-The algorithm for the proposed compact active sound absorption system.}

Calibration before the control

1. Excite the secondary source using a modeling signal $s(n)$, and capture the signals picked up by the sensors A, B, and C, i.e., $p_{\mathrm{A}}(n), p_{\mathrm{B}}(n)$, and $p_{\mathrm{C}}(n)$, simultaneously. The sound pressure due to the $(0,0)$ mode $p_{\mathrm{E}(0,0)}(n)$ can be calculated from $p_{\mathrm{C}}(n)$ with $\left\{w_{M-\tau f s}(n)\right\}$ by using Eqn. (7).

2. The equivalent impulse response $\left\{w_{\mathrm{SE}(0,0)}(n)\right\}$ between the secondary source and $p_{\mathrm{E}(0,0)}(n)$, the impulse response $\left\{w_{\mathrm{SA}}(n)\right\}$ between the secondary source and $p_{\mathrm{A}}(n)$ and the impulse response $\left\{w_{\mathrm{SB}}(n)\right\}$ between the secondary source and $p_{\mathrm{B}}(n)$ are estimated by using the LMS algorithm.

Controlling process

1. The sound pressure related only to the far-field primary source at sensors A and B is calculated by Eqn. (9).

2. The incident and reflected waves related only to the primary source at position E are calculated by Eqns. (10) and (11). The sound pressure due to the $(0,0)$ mode contributed only by the secondary source at position $\mathrm{E}$ is obtained by Eqn. (12).

3. The incident wave $p_{\mathrm{iE}(0,0)}(n)$ and the reflected wave $p_{\mathrm{rE}(0,0)}(n)$ at position $\mathrm{E}$ are calculated by Eqns. (13) and (14).

4. $p_{\mathrm{iE}(0,0)}(n)$ is set as the reference signal, $p_{\mathrm{rE}(0,0)}(n)$ as the target signal, and $\left\{w_{\mathrm{SE}(0,0)}(n)\right\}$ as the secondary path, and then $p_{\mathrm{rE}}(n)$ is attenuated by using the FxLMS algorithm. 


$$
p_{\mathrm{E}(0,0)}^{\mathrm{s}}(n)=\sum_{l=0}^{L_{\mathrm{SE}(0,0)}-1} w_{\mathrm{SE}(0,0)}(l) s(n-l) .
$$

Finally, the plane wave component from the incident and reflected waves at position $\mathrm{E}$ can be obtained by:

$$
\begin{gathered}
p_{\mathrm{iE}(0,0)}(n)=p_{\mathrm{iE}}^{\mathrm{p}}(n), \\
p_{\mathrm{rE}(0,0)}(n)=p_{\mathrm{rE}}^{\mathrm{p}}(n)+p_{\mathrm{E}(0,0)}^{\mathrm{s}}(n) .
\end{gathered}
$$

The proposed algorithm with the calibration process and the controlling process is summarized in Table 1, where the calibration filters, $\left\{w_{\mathrm{SE}(0,0)}(n)\right\},\left\{w_{\mathrm{SA}}(n)\right\}$ and $\left\{w_{\mathrm{SB}}(n)\right\}$, are obtained in the calibration process before the control.
In the controlling process, only sensors A and B are required while the sensor $C$ is removed. Therefore, the control system is compact while the near-field effect of the secondary source is eliminated. The limitations of the proposed algorithm are that the sound field (the pre-identified transfer functions) of the system must be stable and the frequencies of the primary sound must be lower than the cutoff frequency of the duct.

\section{EXPERIMENTS}

In the experiment system depicted in Fig. 2, the 325-cmlong duct has a cross section size of $17 \mathrm{~cm} \times 17 \mathrm{~cm}$ with a cut-off frequency of about $1000 \mathrm{~Hz}$. The primary source and sound-absorbing material are placed at the left end of the duct so that the noise can be regarded as incident from

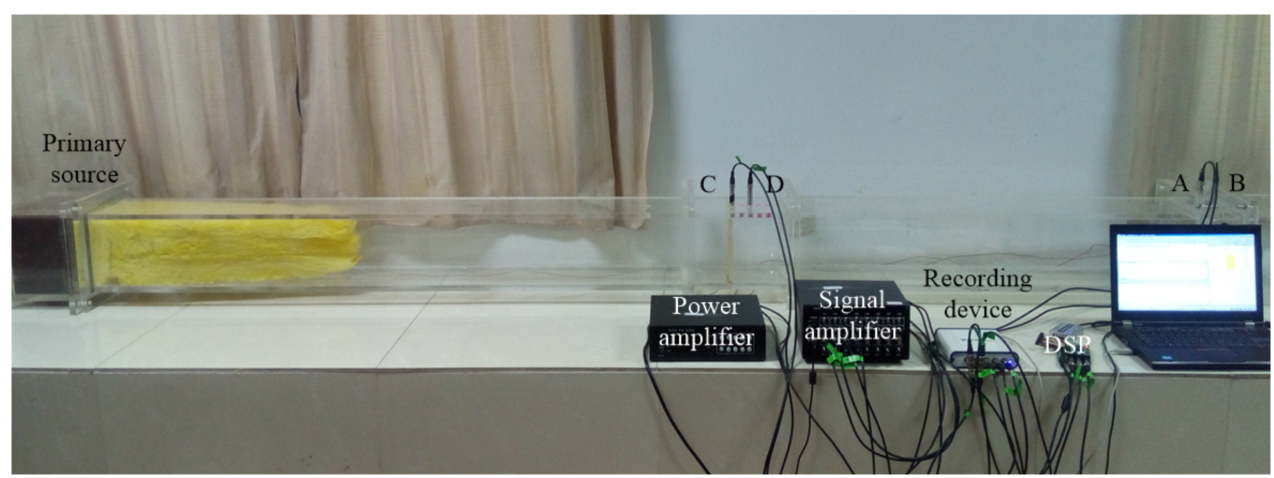

$\mathrm{a}$

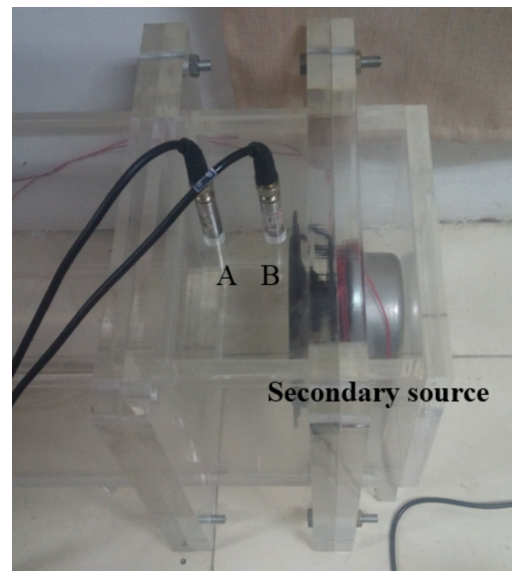

b

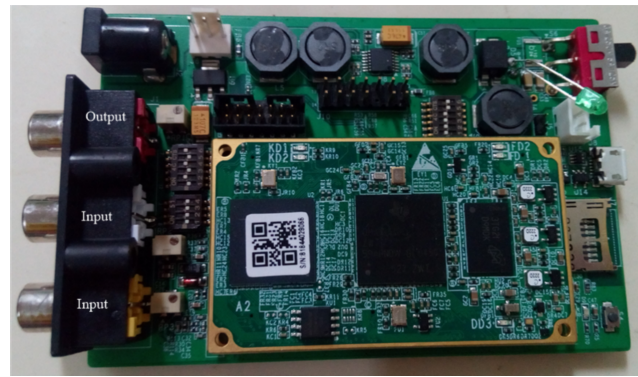

c

Fig. 2-Experimental configurations with (a) The whole system, (b) The secondary source and the sensors and (c) The controller hardware. 
Table 2-Wave separation results with the secondary source excited by sinusoidal signal.

\begin{tabular}{|c|c|c|c|c|c|c|c|c|}
\hline & \multirow{2}{*}{$\frac{\text { Wave separation }}{\text { Frequency }(\mathrm{Hz})}$} & \multicolumn{7}{|c|}{ Amplitude } \\
\hline & & 300 & 400 & 500 & 600 & 700 & 800 & 900 \\
\hline \multirow[t]{2}{*}{ Sensors A and B } & Wave in the $\mathrm{z}$ - direction & 0.32 & 0.35 & 0.27 & 0.31 & 0.20 & 0.21 & 0.13 \\
\hline & Wave in the $\mathrm{z}+$ direction & 0.57 & 0.56 & 0.51 & 0.99 & 0.55 & 0.55 & 0.58 \\
\hline \multirow[t]{2}{*}{ Sensors C and D } & Wave in the $\mathrm{z}-$ direction & 0.08 & 0.07 & 0.07 & 0.16 & 0.08 & 0.08 & 0.05 \\
\hline & Wave in the $\mathrm{z}+$ direction & 0.24 & 0.28 & 0.33 & 0.84 & 0.49 & 0.50 & 0.51 \\
\hline
\end{tabular}

far-field. The secondary source is placed at the right end of the duct. Note that a thinner secondary loudspeaker can be utilized and the size of the control system can be further constrained. Sensor A is placed less than $5.0 \mathrm{~cm}$ to the secondary source to form a compact system, and the interval between the sensor A and the sensor B is $4.0 \mathrm{~cm}$. The distances from the calibration sensors $\mathrm{C}$ and $\mathrm{D}$ to both duct ends exceed $110 \mathrm{~cm}$. The algorithm described in Table 1 is implemented in a real time ANC controller using a TMS320C6747 DSP processer with the sampling rate $f_{s}=16 \mathrm{kHz}^{19}$. The normally used algorithm without the calibration process is also implemented in the DSP platform for comparison purpose. To guarantee a proper calibration and control process, all the related parameters are chosen carefully to make sure that the system is stable without the influence of the near-field effect.

To demonstrate the influence of the near-field effect, the secondary source is excited and the signals received by two groups of the sensors are used to separate the acoustic wave into the incident and reflected signals. Sensors C and D are placed farther away from the end of the duct, so high-order mode waves barely influence the plane wave transmission at positions $\mathrm{C}$ and $\mathrm{D}$. Therefore, the incident and reflected plane waves separated from the signals received by sensors $\mathrm{C}$ and $\mathrm{D}$ are reasonably good. In Table 2, the amplitude of the plane wave in $z+$ direction is much smaller than that in $z$ - direction calculated from the signals captured by sensors $\mathrm{C}$ and $\mathrm{D}$ due to the sound absorption from the wedge installed at the other end of the duct. The experiment results in Table 2 show that the wave separation results at sensors A and B are significantly deviated from those at sensors $\mathrm{C}$ and D. To further manifest the near-field effect, the measured wave amplitude at position B is compared

Table 3-The wave amplitude at position B with secondary source excited by sinusoidal sound.

\begin{tabular}{lccc}
\hline \hline & $\begin{array}{c}\text { Frequency } \\
(\mathrm{Hz})\end{array}$ & $p_{\mathrm{B}}(n)$ & $p_{\mathrm{B}(0,0)}(n)$ \\
\hline Amplitude & 400 & 0.34 & 0.23 \\
& 600 & 0.71 & 0.99 \\
& 800 & 0.38 & 0.44 \\
\hline \hline
\end{tabular}

with the expected plane wave amplitude when the secondary source is excited by sinusoidal signals, as shown Table 3 .

Table 4 shows the measured sound absorption coefficients from sensors $\mathrm{C}$ and $\mathrm{D}$ with the active control system. It can be seen that the proposed algorithm can increase the acoustic absorption effectively at all frequencies. The performance deteriorates significantly and the system is even unstable without the proposed calibration process. For sinusoidal sound control results given in Table 4, the length of all filters is 20 .

To further investigate the performance of the compact active control system, the control of the narrow-band noise signal is also carried out. The frequency range of the narrowband noise is from 700 to $800 \mathrm{~Hz}$. The length of all filters is 90. The absorption coefficients of the reflector before and after control are shown in Fig. 3. It can be seen that the absorption coefficient of the reflector increases significantly using the improved control method. It should be noted that the whole compact system is expected to be causal, but the delay caused by the $\mathrm{AD} / \mathrm{DA}$ converters in the hardware, which is about $200 \mu \mathrm{s}$, undermines the causality of the system $^{20}$. The causality of the system can be guaranteed if some ultra-fast $\mathrm{AD} / \mathrm{DA}$ converters can be used.

Note that when the active absorbing system is used in a free field or used as a side-branch of the duct, the near-field effect will change. For side-branch implementation, the calibration process with auxiliary sensors is similar to the one proposed in this paper. The control of the reflected

Table 4-The sound absorption coefficients measured from sensors $C$ and $D$.

\begin{tabular}{lccc}
\hline \hline $\begin{array}{l}\text { Frequency } \\
(\mathrm{Hz})\end{array}$ & Before control & \multicolumn{2}{c}{ After control } \\
\cline { 3 - 4 } & & $\begin{array}{c}\text { Without } \\
\text { calibration }\end{array}$ & $\begin{array}{c}\text { With } \\
\text { calibration }\end{array}$ \\
\hline 300 & 0.05 & 0.45 & 0.98 \\
400 & 0.09 & 0.78 & 1.00 \\
500 & 0.19 & 0.83 & 1.00 \\
600 & 0.28 & 0.80 & 0.99 \\
700 & 0.36 & 0.93 & 1.00 \\
800 & 0.37 & 0.93 & 1.00 \\
900 & 0.36 & Unstable & 1.00 \\
\hline \hline
\end{tabular}




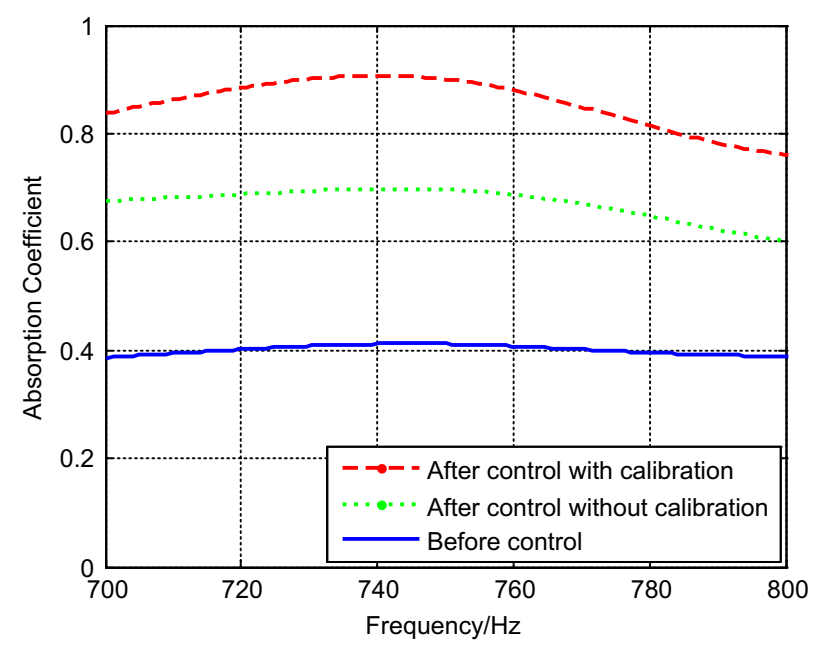

Fig. 3-The absorption coefficient of the reflector tested from the narrow-band signal before and after control.

wave in a free field is a big and unresolved challenge, and the near-field effect is still unavoidable as long as the sensors are placed very close to the control sources.

\section{CONCLUSIONS}

The performance of active sound absorption using the compact control system deteriorates considerably in a duct due to the near-field effect of the secondary source. An improved active control method is proposed by introducing an effective calibration process before control. The extra sensor is only required at the calibration process, and the whole control system is kept in a compact form during the control process. The experiments show that the sound absorption coefficients increase significantly in a wide range up to the cut-off frequency using the improved control method.

\section{ACKNOWLEDGMENTS}

We are sincerely grateful to the anonymous reviewers whose comments help us improve the quality of the paper significantly. This work was supported by National Natural Science Foundation of China (Grant No. 11374156 and No. 11474163) and the Fundamental Research Funds for the Central Universities.

\section{REFERENCES}

1. J.J. Finneran and M.C. Hastings, "Active impedance control within a cylindrical waveguide for generation of low-frequency, underwater plane traveling waves", J. Acoust. Soc. Am., 105(6), 3035-3043, (1999).

2. M.A. Galland, B. Mazeaud and N. Sellen, "Hybrid passive/active absorbers for flow ducts", Appl. Acoust., 66(6), 691-708, (2005).

3. J.B. Dupont and M.A. Galland, "Active absorption to reduce the noise transmitted out of an enclosure", Appl. Acoust., 70(1), 142-152, (2009).

4. N. Han and C. Wang, "Time-domain simulation for active noise control in a two dimensional duct", Noise Control Engr. J., 63(1), 59-71, (2015)

5. M. Furstoss, M.A. Galland and D. Thenail, "Surface impedance control for sound absorption: direct and hybrid passive/active strategies", J. Sound Vib., 203(2), 219-236, (1997).

6. J.P. Smith, B.D. Johnson and R.A. Burdisso, "A broadband passiveActive sound absorption system", J. Acoust. Soc. Am., 106, 2646-2652, (1999).

7. N. Sellen, M. Cuesta and M.A. Galland, "Noise reduction in a flow duct: Implementation of a hybrid passive/active solution", J. Sound. Vib., 297(3), 492-511, (2006).

8. P. Cobo and M. Cuesta, "Hybrid passive-active absorption of a microperforated panel in free field conditions", J. Acoust. Soc. Am., 121, 251-255, (2007).

9. N. Han and J. Tao, "Unidirectional acoustic boundary through active control system in one dimensional duct", Noise Control Engr. J., 63(5), 388-395, (2015).

10. P. Cobo and M. Cuesta, "Measuring hybrid passive-active sound absorption of a microperforated liner at oblique incidence", $J$. Acoust. Soc. Am., 125, 185-190, (2009).

11. N. Han, S. Feng and X. Qiu, "Active control of one-dimension impulsive reflection based on a prediction method", J. Acoust. Soc. Am., 127(3), 1193-1196, (2010).

12. N. Han, X. Qiu and S. Feng, "Active control of three-dimension impulsive scattered radiation based on a prediction method", Mechanical Systems and Signal Process., 30, 267-273, (2012).

13. H. Zhu, R. Rajamani and K.A. Stelson, "Active control of acoustic reflection, absorption, and transmission using thin panel speakers", J. Acoust. Soc. Am., 113(2), 852-870, (2003).

14. Acoustics - Determination of sound absorption coefficient and impedance in impedance tubes-part 2: Transfer function method, BS EN ISO 10534-2: 1990, International Organization for Standardization, Switzerland, (1998).

15. W.H. Press, S.A. Teukolsky, W.T. Vetterling and B.P. Flannery, in Numerical Recipes 3rd Edition: The Art of Scientific Computing, Chap. 4, Cambridge University Press, New York, pp. 155-162, (2007).

16. P.A. Nelson and S.J. Elliott, in Active Control of Sound, Chap. 6, Academic Press, San Diego, pp. 195-198, (1991).

17. P.M. Morse and K.U. Ingard, in Theoretical Acoustics, Chap. 9, Princeton University Press, New Jersey, pp. 492-498, (1968).

18. T.I. Laakso, V. Valimaki, M. Karjalainen and U.K. Laine, "Splitting the unit delay", IEEE Signal Process. Mag., 13(1), 30-60, (1996).

19. TMS320C6745, TMS320C6747 Fixed- and Floating-Point Digital Signal Processor (2014), http://www.ti.com/lit/ds/symlink/ tms320c6747.pdf, Accessed on 9 March 2017.

20. L. Zhang and X. Qiu, "Causality study on a feedforward active noise control headset with different noise coming directions in free field", Appl. Acoust., 80, 36-44, (2014). 
Copyright of Noise Control Engineering Journal is the property of Institute of Noise Control Engineering of the USA and its content may not be copied or emailed to multiple sites or posted to a listserv without the copyright holder's express written permission. However, users may print, download, or email articles for individual use. 